\title{
Tubers from patients with tuberous sclerosis complex are characterized by changes in microtubule biology through ROCK2 signalling
}

\author{
Isidre Ferrer,"\# Pooja Mohan, ${ }^{2 \#}$ Helen Chen, ${ }^{2}$ Joan Castellsague, ${ }^{3}$ Laia Gómez-Baldó, ${ }^{4}$ Marga Carmona, \\ Nadia García, ${ }^{4}$ Helena Aguilar, ${ }^{4}$ Jihong Jiang, ${ }^{2}$ Margaretha Skowron,, Mark Nellist, ${ }^{4}$ Israel Ampuero, ${ }^{6}$ Antonio \\ Russi, ${ }^{7}$ Conxi Lázaro, ${ }^{3}$ Christopher A Maxwell2* ${ }^{2 *}$ and Miguel Angel Pujana ${ }^{4 *}$ \\ I Institute of Neuropathology, University Hospital Bellvitge, University of Barcelona, Bellvitge Institute for Biomedical Research (IDIBELL), \\ CIBERNED, L'Hospitalet del Llobregat, Barcelona, Catalonia, Spain \\ 2 Department of Pediatrics, University of British Columbia, Child and Family Research Institute, Vancouver, BC, Canada \\ 3 Hereditary Cancer Programme, Catalan Institute of Oncology (ICO), IDIBELL, L'Hospitalet del Llobregat, Barcelona, Catalonia, Spain \\ 4 Breast Cancer and Systems Biology Unit, Translational Research Laboratory, ICO, IDIBELL, L'Hospitalet del Llobregat, Barcelona, Catalonia, \\ Spain \\ 5 Department of Clinical Genetics, Erasmus Medical Centre, Rotterdam, The Netherlands \\ 6 Banco de Tejidos para Investigaciones Neurológicas, Facultad de Medicina, Universidad Complutense de Madrid, Spain \\ 7 Epilepsy Unit, Teknon Medical Centre, Barcelona, Catalonia, Spain
}

\begin{abstract}
*Correspondence to: CA Maxwell, Department of Pediatrics, University of British Columbia, Child and Family Research Institute, 950 28th Avenue West, Vancouver, BC V5Z 4H4, Canada. E-mail: cmaxwell@cfri.ca

Or Dr. Miguel Angel Pujana, Breast Cancer and Systems Biology Unit, Translational Research Laboratory, ICO, IDIBELL, L'Hospitalet del Llobregat, Barcelona, Catalonia, Spain. Email: mapujana@iconcologia.net
\end{abstract}

\#These authors contributed equally to this study.

\begin{abstract}
Most patients with tuberous sclerosis complex (TSC) develop cortical tubers that cause severe neurological disabilities. It has been suggested that defects in neuronal differentiation and/or migration underlie the appearance of tubers. However, the precise molecular alterations remain largely unknown. Here, by combining cytological and immunohistochemical analyses of tubers from nine TSC patients (four of them diagnosed with TSC2 germline mutations), we show that alteration of microtubule biology through ROCK2 signalling contributes to TSC neuropathology. All tubers showed a larger number of binucleated neurons than expected relative to control cortex. An excess of normal and altered cytokinetic figures was also commonly observed. Analysis of centrosomal markers suggested increased microtubule nucleation capacity, which was supported by the analysis of an expression dataset from cortical tubers and control cortex, and subsequently linked to under-expression of Rho-associated coiled-coil containing kinase 2 (ROCK2). Thus, augmented microtubule nucleation capacity was observed in mouse embryonic fibroblasts and human fibroblasts deficient in the Tsc2/TSC2 gene product, tuberin. Consistent with ROCK2 under-expression, microtubule acetylation was found to be increased with tuberin deficiency; this alteration was abrogated by rapamycin treatment and mimicked by HDAC6 inhibition. Together, the results of this study support the hypothesis that loss of TSC2 expression can alter microtubule organization and dynamics, which, in turn, deregulate cell division and potentially impair neuronal differentiation.

Copyright @ 2014 Pathological Society of Great Britain and Ireland. Published by John Wiley \& Sons, Ltd.
\end{abstract}

Keywords: cytokinesis; microtubule; ROCK2; TSC2; tuberous sclerosis complex

Received 17 September 2013; Revised 20 January 2014; Accepted 28 February 2014

No conflicts of interest were declared.

\section{Introduction}

Heterozygous loss-of-function mutations in the tumour suppressor genes TSC1 and TSC2 cause the autosomal dominant disorder tuberous sclerosis (TSC) [1]. TSC patients present multi-system pathology characterized by abnormal cell growth and proliferation. Cortical tubers are present in $>80 \%$ of patients and are manifested by the presence of dysplastic neurons, glial cells and glioneuronal giant cells, which together disrupt the laminar architecture of the cortex [2]. As a result, TSC patients often suffer from severe epilepsy, mental retardation, autism and/or attention deficit hyperactivity disorder [3]. 
Hamartin and tuberin, the respective products of the TSC1 and TSC2 genes, form a heterodimer that inhibits the mechanistic target of rapamycin (mTOR) complex 1 (mTORC1) [4]. Mutations in TSC1 or TSC2 lead to an increase in the activity of the RAS homologue enriched in brain (RHEB), which, in turn, activates mTORC1 [4]. Downstream targets of mTORC1 include the ribosomal protein S6 kinase and the eukaryotic translation initiation factor $4 \mathrm{E}$ binding protein 1 [4]. The hamartin-tuberin heterodimer also activates mTOR complex 2 (mTORC2), which positively regulates the actin cytoskeleton [4].

It has been proposed that the neurological alterations of TSC are caused by defects in neuronal differentiation and/or migration $[5,6]$. Depletion of hamartin or tuberin in mice was shown to induce ectopic axons and promote axon growth, leading to perturbed neuronal polarity [7]. More recent studies that examined the phenotypic consequences of hamartin or tuberin depletion in neuronal cell types, including neuronal stem cells, have further suggested defects in polarity, differentiation and/or migration [8-11]. In addition, studies of different cell types have linked loss of function of hamartin or tuberin to defects in primary cilium formation, cell polarity and division [12-16]. This array of cellular defects may indicate a deregulation of the microtubule cytoskeleton and/or its organization and assembly at the centrosome, the major microtubule-organizing centre in dividing animal cells. Moreover, hamartin was previously shown to physically interact with polo-like kinase 1 (PLK1) [13], a key kinase regulator in microtubule assembly, while tuberin functionally associates with the microtubule-associated protein TACC3 in maintaining the integrity of the mitotic spindle and nuclear envelope [12]. To date, however, detailed examination of microtubule-centred cellular and molecular alterations is lacking in TSC.

\section{Materials and methods}

\section{Tissue and genetic analyses}

Brain tissues from three unrelated controls and nine TSC patients were obtained at the Institute of Neuropathology (University Hospital Bellvitge, IDIBELL, L'Hospitalet del Llobregat, Barcelona) and at the Epilepsy Unit of the Teknon Medical Centre (Barcelona). Participants provided written informed consent and the study was approved by the IDIBELL's ethics committee. All clinical investigation was conducted according to the principles expressed in the Declaration of Helsinki. Tissues were obtained at surgery, fixed in $10 \%$ buffered formalin and processed for paraffin sections. Sections were stained with haematoxylin and eosin (H\&E), following a standard protocol, and histopathologically diagnosed at the Institute of Neuropathology. Genetic analyses were performed at the Centre for Molecular Diagnostics of Neurological Diseases of the Universidad Complutense (Madrid) and at the Department of Clinical Genetics of the Erasmus Medical Centre (Rotterdam).

\section{Antibodies, small molecules} and immunohistochemistry

The antibodies used in this study were: anti-centriolar coiled-coil protein, $110 \mathrm{kDa}$ (CP110) [17]; anti-nucleoporin, $62 \mathrm{kDa}$ (NUP62; clone 53, BD Transduction Laboratories); anti-PLK1 (clone 208G4, Cell Signaling Technology); anti-phospho-Ser235-236 S6 ribosomal protein (pS6; clone 91B2, Cell Signaling Technology); anti-ROCK2 (clone 21, BD Transduction Laboratories); anti-TACC3 (H-300, Santa Cruz Biotechnology); anti- $\alpha$-tubulin (TUBA; ab44928, Abcam); anti-acetylated tubulin (acTUB; clone 6-11B-1, Sigma Aldrich); and anti- $\gamma$-tubulin (TUBG1; clone GTU-88, Sigma-Aldrich). Rapamycin (allosteric mTOR inhibitor, purchased from Sigma-Aldrich) and CAY10603 (HDAC6-specific inhibitor, purchased from Santa Cruz Biotechnology) were added to cell cultures at 44 and 2 $\mathrm{nM}$, respectively, for $16 \mathrm{~h}$ prior to analysis of microtubule acetylation levels. Immunohistochemical analyses were performed on serial paraffin sections, $4 \mu \mathrm{m}$ thick, using the Envision method (Dako). The antibody dilutions and antigen retrieval-based solutions (dilution/solution) were: 1:1000/EDTA, 1:300/EDTA, 1:100/sodium citrate, 1:500/sodium citrate, 1:300/EDTA, 1:300/sodium citrate and 1:1000/EDTA for anti-CP110, anti-NUP62, anti-PLK1, anti-pS6, anti-ROCK2, anti-TACC3 and anti-TUBG1, respectively. Endogenous peroxidase was blocked by pre-incubation in a solution of $3 \% \mathrm{H}_{2} \mathrm{O}_{2}$, and blocking was performed in $1 \times$ phosphate-buffered saline (PBS) with $5 \%$ goat serum or $1 \%$ bovine serum albumin (BSA) and $0.1 \%$ Tween 20 (Sigma-Aldrich). The sections were lightly counterstained with haematoxylin (except for anti-NUP62, to allow nuclei assessment), and examined with an Olympus BX51 microscope. Detection of ROCK2 was carried out using the DAB-nickel method. The specificity of the anti-TUBG1 antibody in paraffin sections was assessed previously [18]. The expected immunostaining pattern of this centrosomal marker in normal cells is one or two foci proximal to the nucleus; nonetheless, its expression in differentiated cells can be low and, thus, hamper the identification of centrosomes. Each tissue sample and marker was evaluated in at least two independent assays and no substantial intratissue difference was observed. For seven TSC tissue samples, equivalent sections were processed to include incubation with a non-immune murine immunoglobulin control, which did not reveal staining in any case. Two investigators carried out quantification of binucleated cells, and cytokinetic figures and abnormalities, independently. Statistical significance was assessed using quadruplicate measures and contingency tables with the Fisher's exact test. 


\section{Silencing of endogenous TSC2 expression and rescue experiments}

The short hairpin (sh) RNA against TSC2 expression (Sigma-Aldrich; for sequence, see supplementary material, Table S1), control non-hairpin pLKO.1 and lentivirus packaging plasmids (Addgene) were as previously described [12]. Transduced cells were selected with $0.5 \mu \mathrm{g} / \mathrm{ml}$ puromycin (GIBCO) and maintained with $0.3 \mu \mathrm{g} / \mathrm{ml}$ puromycin. The assays assessing the consequences of shRNA-mediated silencing of TSC2 expression in fibroblasts represented a pooled population of puromycin-resistant cells. The small interfering (si) RNAs against TSC2 expression (Dharmacon; for sequence, see supplementary material, Table S1) and control scrambled siRNA were transfected into cells as previously described [19]. For the reconstitution assays, cells were transiently transfected with GFP-TSC2, GFP-TSC2 and TSC1-His or GFP-alone expression constructs, using Lipofectamine. Cellular phenotypes were measured in GFP-positive cells.

\section{Quantitative reverse transcriptase - polymerase chain reaction}

RNA extraction from human BJ fibroblasts and sub-lines, and quantitative reverse transcriptionpolymerase chain reactions (qRT-PCRs) were performed as described previously [19]. The primers used in these assays are detailed in Table S1 (see supplementary material).

\section{Microtubule regrowth assay and acetylation analysis}

The $T s c 2^{+/+} / T p 53^{-/-}$and Tsc2 $2^{-/-} / T p 53^{-/-}$mouse embryonic fibroblasts (MEFs) and the human foreskin BJ fibroblasts (ATCC) and sub-lines were cultured following standard protocols. Cells growing on coverslips were treated with $1 \mu \mathrm{M}$ nocodazole at $37^{\circ} \mathrm{C}$ for $16 \mathrm{~h}$, followed by drug washout and incubation on ice (cold medium) for $1 \mathrm{~h}$. Cells were then recovered in pre-warmed growth medium at $37^{\circ} \mathrm{C}$ to allow microtubule regrowth, and subsequently fixed (in cold methanol) at various time points for immunostaining of TUBA. Microtubule regrowth was analysed in mitotic cells, as indicated by DNA condensation. Microtubule asters were quantified using maximal projections derived from ImageJ (National Institutes of Health, Bethesda, MD, USA). For analysis of acetylated tubulin (acTUB), cells growing on coverslips were stained for acTUB and total tubulin (TUBA) and counterstained with DAPI. Confocal images were acquired and the levels of acTUB were normalized to the intensity of TUBA, using FV10-ASW software.

\section{Gene set expression analysis}

Preprocessed and normalized microarray data for control cortex and cortical tubers were obtained from the Gene Expression Omnibus reference GSE16969
[20]. This dataset included gene expression analysis for tissues from four normal cortex and four cortical tubers (two cases with a mutation in TSC1 and two cases with a mutation in TSC2) [20]. The distribution of Gene Ontology (GO) [21] gene sets in whole-genome ranked GSE16969 results was examined using the gene set expression analysis (GSEA) [22] tool, with default values for all parameters. This analysis assessed the bias in the distribution of each GO term (linked to genes) in the whole-genome ranking of average gene expression differences between the cortical tuber and normal cortex tissue.

\section{Results}

\section{Binucleated neurons in tubers}

Cortical tubers from nine TSC patients and cortex tissue from three unrelated healthy individuals were examined in this study. Patients were clinically diagnosed with TSC following consensus diagnostic criteria. As part of their clinical evaluation, four patients were identified as carrying heterozygous germline mutations in TSC2; the remaining three were not characterized at the genetic level (see supplementary material, Table S2). According to the diagnoses, all tubers showed the classic histopathological features of TSC, which include disorganization of the cortical layer, increased astrocytic gliosis and the presence of large binucleated cells (Figure 1A; see also supplementary material, Figure S1). Quantification of binucleated cells revealed a significantly higher percentage in all tubers relative to controls (Fisher's exact test $p$ values $<0.01$; Figure 1B). This observation is consistent with previous neurohistological studies [2] and with cellular analyses of MEFs deficient in tuberin [12]. Hamartin deficiency was also previously linked to an increase in binucleated MEFs [12]. As expected, enhanced activation of mTORC1 signalling (revealed by pS6 staining) was detected in all tubers examined in this study and was particularly evident in giant cells (Figure 1A; see also supplementary material, Figure S1).

\section{Cytokinesis alteration in tubers}

Among the potential defects in cell division, failure of cytokinesis may be the cause of the binucleated cells [23]. Previously, deficiency of hamartin or tuberin in MEFs was also linked to a delay in completion of cytokinesis and, by extension, to aberrant abscissions [12]. Frequent cytokinesis failure was also observed in cell lines derived from skin angiofibromas of TSC patients [24]. Therefore, we subsequently examined H\&E-stained cortical tuber sections for the proportion of cytokinetic figures and potential associated alterations. All tubers revealed a higher number of cytokinetic figures (most of them apparently aberrant) than expected, relative to control tissue (Figure 2A, 
A
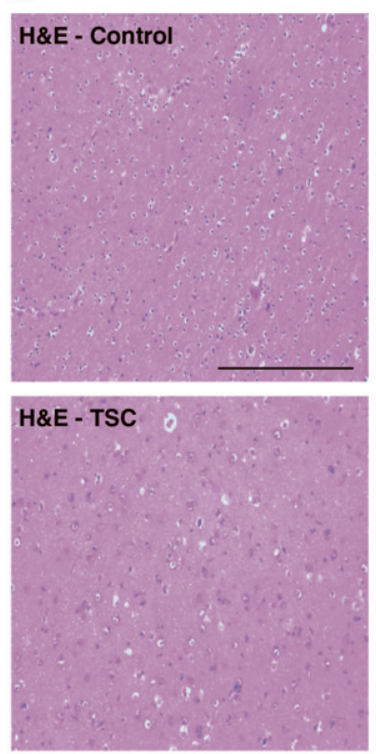
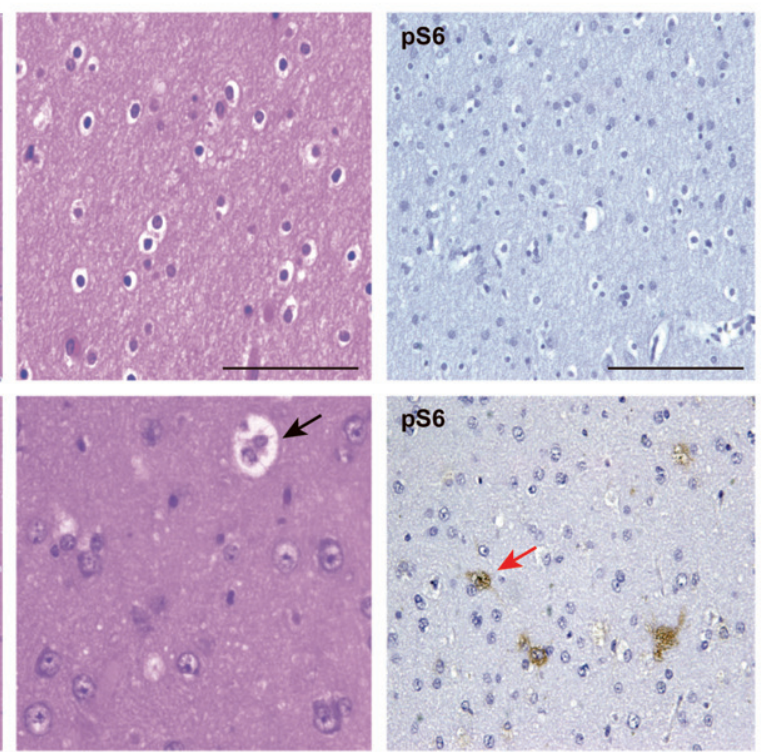

B

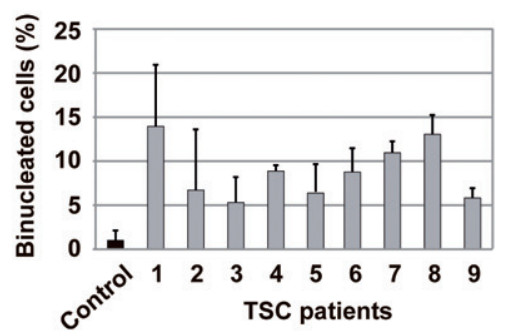

Figure 1. Binucleated cells and pS6 positivity in cortical tubers. (A) Representative images of H\&tE staining (left and middle panels) and pS6 immunostaining (right panels) in a control cortex and in a TSC tuber (images for all tubers are provided in Figure S1). A black-filled arrow indicates a binucleated cell and a red-filled arrow indicates cellular pS6 positivity; scale bars = (left) $400 \mu$ m; (middle) $200 \mu \mathrm{m}$; (right) 300 $\mu \mathrm{m}$. (B) Quantification (represented as percentage) of binucleated cells in the control sample and tubers. The results correspond to four randomly chosen fields with at least 50 cells in each, examined in each sample; error bars show SD for the measured fields

quantification provided in panel $2 \mathrm{~B}$; see also supplementary material, Figure S2); consequently, aberrantly shaped nuclei were frequently observed (Figure S2). Next, immunohistochemical detection of the nucleoporin NUP62 confirmed the presence of cytokinetic abnormalities (Figure 2C shows representative images of control and tuber tissue).

\section{Alteration of TUBG1 in tubers}

Cytokinesis failure may be the consequence or cause of abnormal centrosome numbers, structure and/or position [25]. Hamartin or tuberin deficiency has been linked to alterations of the centrosome, such as amplification in hamartin-deficient cells [13], and of centrosome-related organelles, such as disrupted primary cilia in hamartinor tuberin-deficient cells [14-16]. On the basis of these observations, the abundance of TUBG1 and the numbers of TUBG1-positive foci (ie potential centrosomes) were evaluated by immunohistochemistry in the tubers. While the majority of cells in control tissues contained one or two signals/cell, which would represent a normal complement of centrosomes, a variety of abnormalities were observed in the tubers, including potential centrosome amplification, fragmentation and hypertrophy, with TUBG1 over-expression revealed in all cases (Figure 3A; see also supplementary material, Figures S3, S4). Quantification indicated a higher number of abnormalities in the tubers than in the controls: all tubers contained $>40 \%$ of cells with TUBG1-related abnormalities (Figure 3B).

We next examined whether the excess TUBG1 immunoreactivity was associated with numerical changes to centriole numbers, which might indicate dysfunction in the control of centrosome duplication [17]. Interestingly, the levels and localization of the centriolar marker CP110 were not substantially altered in any of the tubers relative to the controls (Figure 3C shows representative images with defined centriolar signals). Taken together, cytological and immunohistochemical studies in tubers from nine TSC patients revealed frequent binucleated cells, cytokinetic abnormalities and augmented TUBG1 abundance without centriole duplication.

\section{Deregulation of proteins involved in microtubule-based processes}

To gain insight into a putative mechanism, whole-genome expression data from cortical tubers and normal cortex [20] were analysed. Consistent with our findings of an excess of binucleated cells (Figure 1) and cytokinetic figures (Figure 2) in tubers, the analysis of genes annotated with the GO term 'Cytokinesis' (genes, $n=47$ ) showed a significant difference in absolute expression values (ie considering expression changes independently of their sign) between tubers and normal cortex $(p=0.038$; Figure 4A). The difference was not significant when real expression values (ie considering the direction of the expression difference, positive or negative) were examined, but an under-expression trend was observed in tubers for genes required for the completion of cytokinesis; the most under-expressed gene in this setting was ROCK2 (Figure 4B).

The under-expression of ROCK2 was of particular interest, given its depicted role in the regulation of 


\section{A}
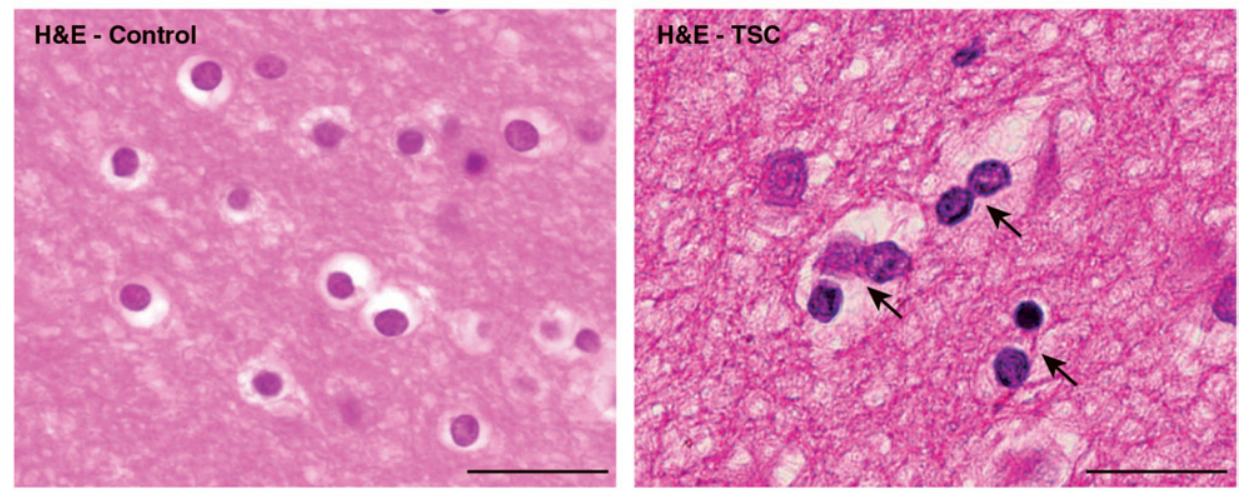

B

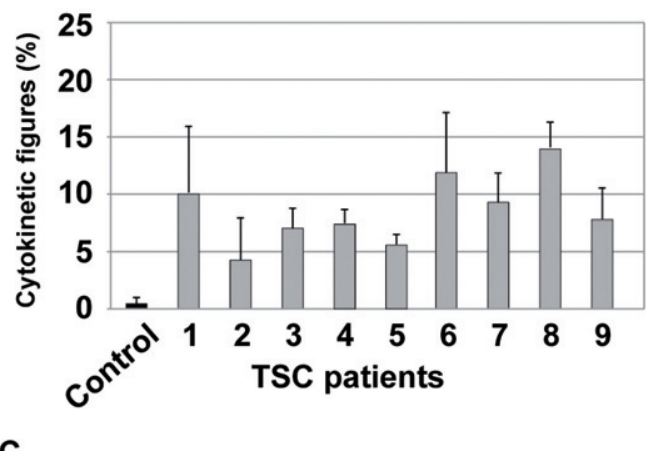

C
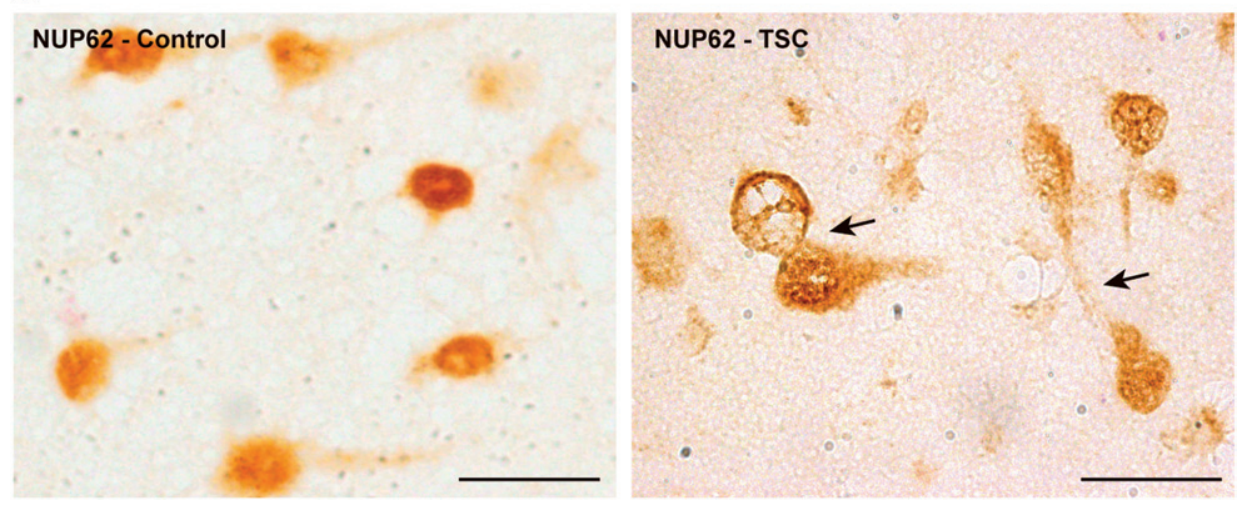

Figure 2. Cytokinesis defects in cortical tubers. (A) Representative images of H\&E-stained control cortex and TSC tuber tissue; arrows indicate cytokinetic figures or alterations in the tuber (images for all tubers are provided in Figure S2, see supplementary material); scale bar $=100 \mu \mathrm{m}$. (B) Quantification (\%) of cytokinetic figures in the control sample and tubers. Results correspond to four randomly-chosen fields examined in each sample; error bars show SD for the measured fields. (C) NUP62 immunostaining in control cortex and TSC tuber tissue; abnormal cytokinetic figures in the tuber are indicated by arrows; scale bar $=50 \mu \mathrm{m}$

microtubule assembly $[26,27]$ as well as in the actomyosin ring contraction required for cleavage furrow ingression and cytokinesis $[28,29]$. Next, immunohistochemical analyses of ROCK2 in our tissue set confirmed its under-expression and association with loss of laminar architecture in the tubers (Figure 4C; see also supplementary material, Figure S5). In addition, the ROCK2 substrate tubulin polymerization-promoting protein 1 (TPPP1) was also suggested to be under-expressed in the analysis of the gene expression dataset (two-tailed $t$-test, $p=0.04)$. The analysis of a gene set corresponding to curated centrosomal proteins [30] also suggested an association with the neuropathology: there was a trend for over-expression of centrosomal genes in tubers relative to normal cortex (real differences GSEA $p=0.022 ;$ and absolute differences GSEA $p=0.046$ ). This association with over-expression of centrosomal gene products may be a consequence of increased cell proliferation, as observed for different malignant neoplasms [31]. Intriguingly, while $P L K 1$ and TACC3 did not contribute to this bias (ie none of their microarray probes showed evidence of differential expression; this was confirmed by immunohistochemistry, not shown), the two probes corresponding to $c h T O G$, which encodes for a key microtubule-binding and TACC-interacting protein [32], indicated significant under-expression in the tubers (two-tailed $t$-test, $p<0.01$ ). According to previous reports [32], loss of chTOG expression may impair microtubule biology and alter cytokinesis. Together, 
A
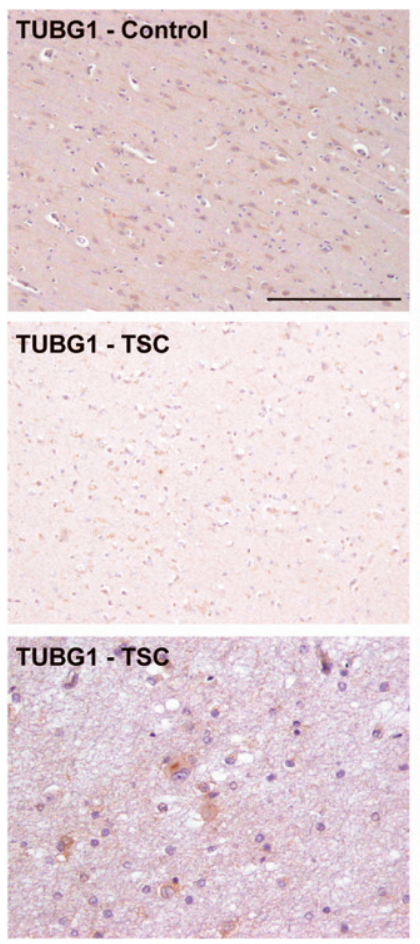
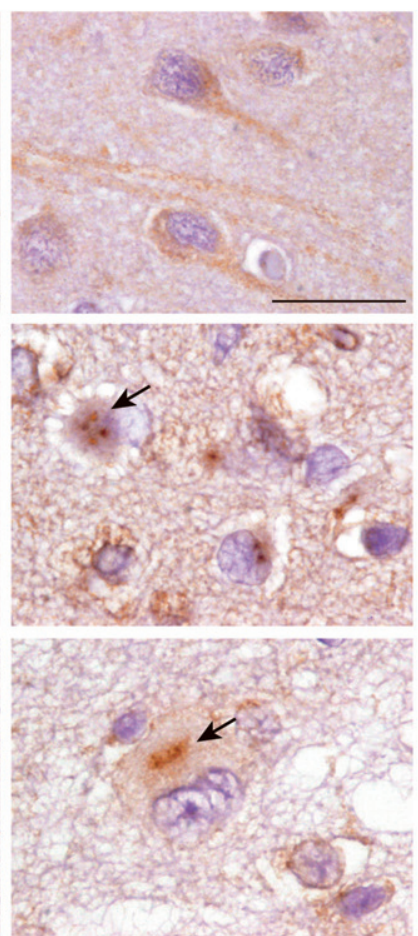

B

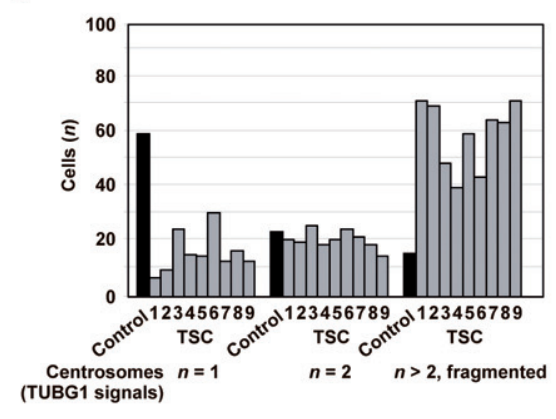

C

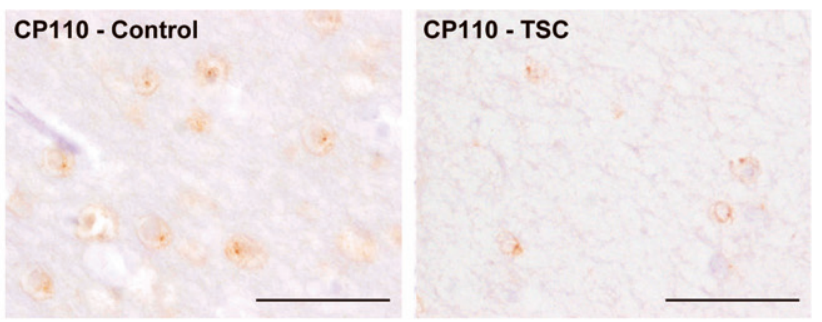

Figure 3. Over-expression and abnormal staining patterns of TUBG1 in cortical tubers. (A) Representative images of TUBG1 immunoreactivity in a control cortex, which shows cytoplasmic staining (top panels), and in TSC tubers (images for all tubers are provided in Figure S3, see supplementary material); arrows indicate abnormal staining or alteration in the expected centrosome number; scale bars $=($ left $) 400 \mu \mathrm{m}$; (right) $50 \mu \mathrm{m}$. (B) Quantification (represented as absolute number, $n$ ) of TUBG1-based signals that could represent centrosomes; the results correspond to at least 200 cells counted in each sample. (C) Representative images of CP110 immunostaining in control cortex and TSC tuber tissue; scale bar $=200 \mu \mathrm{m}$

these observations further suggest an underlying alteration of microtubule biology in TSC neuropathology and, specifically, point to loss of ROCK2 function as a critical factor in this context.

\section{Increased microtubule regrowth with deficiency of tuberin}

To test our hypothesis that loss of TSC2 expression leads to increased microtubule regrowth capacity, we first examined a murine cell model of tuberin deficiency, within which we previously characterized abscission alterations and increased frequency of binucleated cells [12]. In synchronous $\mathrm{G}_{2} / \mathrm{M}$ populations of $T s c 2^{+/+} / T p 53^{-/-}$and $T s c 2^{-/-} / T p 53^{-/-}$MEFs, quantitation of microtubule regrowth (ie the length of microtubules in asters) was found to be significantly greater in tuberin-deficient MEFs than in controls ( $t$-test, $p<0.05$; Figure 5A, B).

Next, we established stable sub-lines of human BJ fibroblasts with lentivirus-mediated transduction of a shRNA targeting TSC2 expression or a non-hairpin-negative shRNA control. A significant reduction in the levels of TSC2 expression was achieved (Figure 6A, B), and this depletion was associated with augmented microtubule regrowth capacity in mitotic cells relative to the control, non-hairpin-treated and untreated control cells (Figure 6C, D). Importantly, we did not observe alterations of the nuclear envelope (as previously reported in MEFs [12]) or gross centrosome abnormalities in non-mitotic tuberin-depleted cells (see supplementary material, Figure S6). Next, we confirmed that augmented microtubule regrowth capacity was the result of the specific loss of TSC2 expression through two complementary approaches: using siRNAs that supported transient loss of TSC2 expression (see supplementary material, Figure S7A, B); and through reconstitution of tuberin expression using transient transfection of a GFP-TSC2 construct, alone or in combination with exogenous hamartin-His (see supplementary material, Figure S7C), which was revealed to be sufficient to recover aster lengths to the levels observed in control shRNA-treated cells (see supplementary material, Figure S7D). Thus, the loss of tuberin expression is sufficient to augment microtubule regrowth capacity, but the mechanism responsible for this effect is not yet clear.

\section{Under-expression of ROCK2 and increased microtubule acetylation}

In tubers relative to normal cortex, both $R O C K 2$ and $T P P P 1$ were predicted to be under-expressed (Figure 4, confirmed for ROCK2 by immunohistochemistry; see also supplementary material, Figure S5). At the molecular level, ROCK2 activity regulates microtubule polymerization through TPPP1 and acetylation through 
A

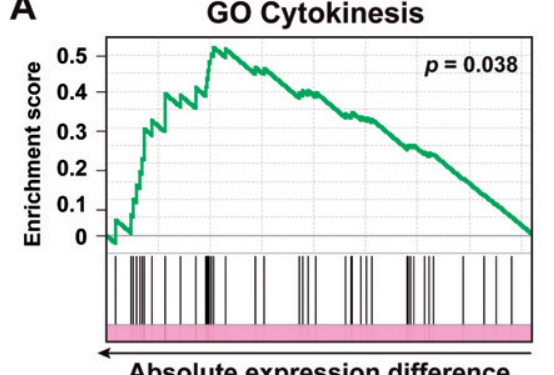

Absolute expression difference (tubers - normal cortex)

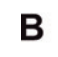

B

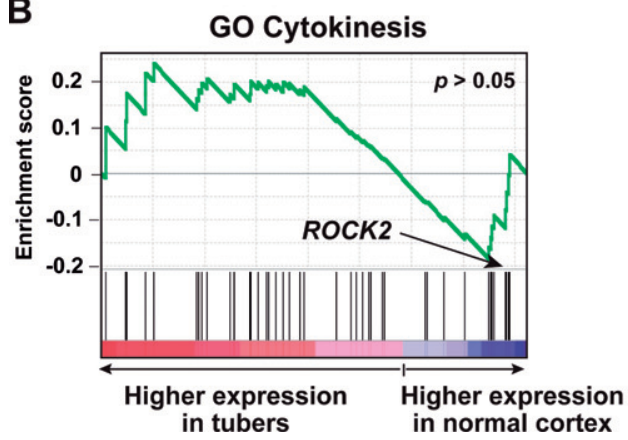

C
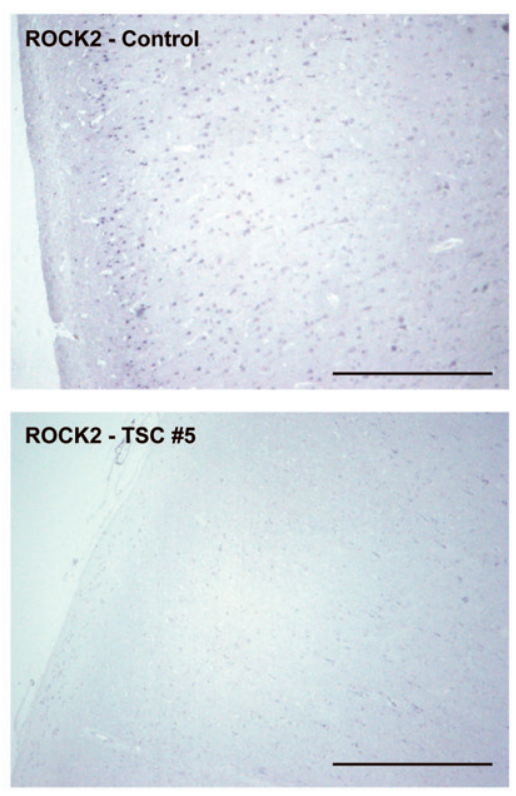
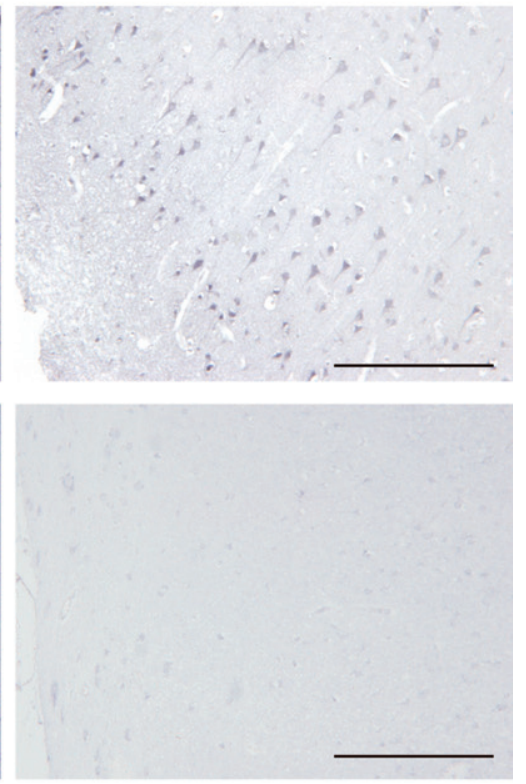

Figure 4. Expression alterations linked to cytokinesis and microtubule-based processes and proteins. (A) Graph showing the GSEA output for the GO 'Cytokinesis' gene set. The enrichment score is shown on the $y$ axis and the gene positions on the $x$ axis, which correspond to the expression differences (in absolute values and taking the maximum difference if $>1$ microarray probes exist for a given gene) between the tuber and normal cortex tissue [20]. The computed $p$ value relative to the enrichment score is shown. (B) Graph showing the GSEA output for the GO 'Cytokinesis' gene set, as above, but ranking expression differences with real values (from positive, which corresponds to higher expression in tubers, to negative, which corresponds to higher expression in normal cortex). (C) Representative images of ROCK2 immunoreactivity in control cortex and TSC tuber tissue (images for all tubers are provided in Figure S5, see supplementary material). The DAB-nickel (blue) method was used in immunodetection; scale bars = (left) $400 \mu \mathrm{m}$; (right) $200 \mu \mathrm{m}$

HDAC6 [26,27]. Next, we investigated the expression of $R O C K 2$ and found it significantly reduced in tuberin-depleted BJ fibroblasts, as measured by qRT-PCR (Figure 7A) and western blot analysis (Figure 7B). Subsequently, microtubule acetylation relative to the total intensity of microtubule immunofluorescence was examined for phenotypic evidence of deregulation of the corresponding signalling pathway. Thus, the levels of acTUB were found to be significantly elevated in tuberin-depleted BJ fibroblasts (Figure 7C, D; see also supplementary material, Figure S8A) and rescue through expression of GFP-tuberin significantly reduced acTUB levels in these cells (see supplementary material, Figure S8B).

Tuberin mutation or depletion leads to an increase in mTORC1 activity [4], so levels of acTUB were subsequently assessed using the mTORC1 inhibitor rapamycin. Exposure to this compound was sufficient to normalize acTUB in tuberin-depleted BJ fibroblasts (Figure 7E). Conversely, the treatment of control cell cultures with a small-molecule-specific HDAC6 inhibitor (CAY10603) was sufficient to elevate acTUB, but the levels of acetylation were not further augmented in tuberin-depleted cells treated with this inhibitor (Figure 7E). Taken together, these data depict a model in which loss of TSC2 expression is sufficient to alter microtubule biology through attenuation of ROCK2 signalling, which culminates in the inhibition of HDAC6 and microtubule acetylation.

\section{Discussion}

In most current models of TSC pathology, loss of tuberin or hamartin results in defects in neuronal migration and differentiation $[7-9,12]$, processes that are expected to be reliant on appropriate microtubule biology [33]. Here, examination of cortical tubers from nine TSC patients suggested that the neuropathology is linked to aberrant microtubule biology, as seen by the increased abundance of binucleated cells, cytokinetic figures and TUBG1 abundance by immunohistochemistry. Gene expression analyses in tubers, as well as in human tuberin-depleted fibroblasts, revealed decreased expression of key components of microtubule biology, particularly of ROCK2. Human tuberin-depleted fibroblasts displayed the expected outcome of diminished ROCK2 expression and increased microtubule acetylation. Thus, attenuated ROCK2 signalling might represent a targetable mechanism for the treatment of TSC tubers. However, given the relatively small sample size in our study, the depicted histopathological alterations may warrant confirmation in larger series of TSC patients. 
A

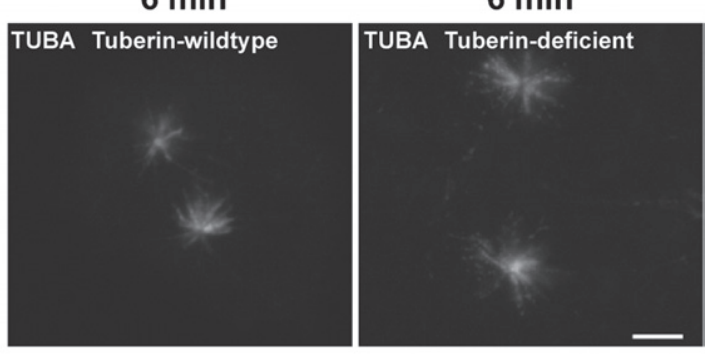

B

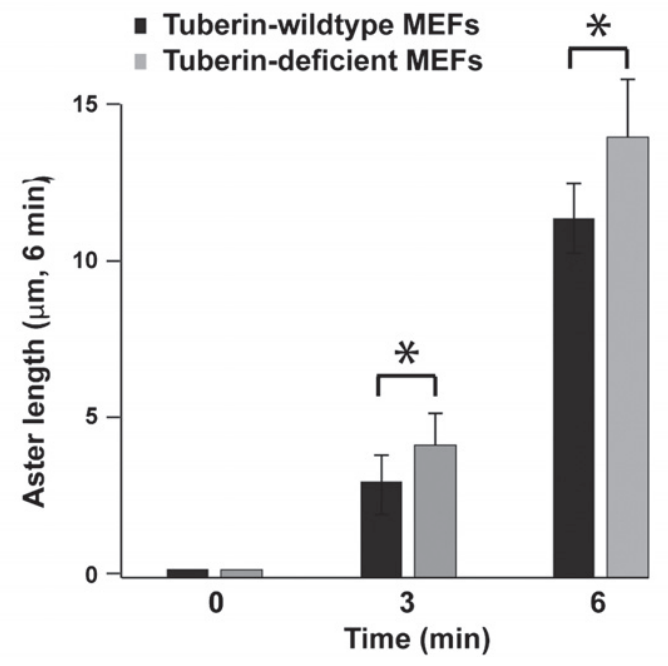

Figure 5. Increased microtubule regrowth capacity in tuberin-deficient MEFs. (A) TUBA immunostaining images for 6 min time points in tuberin-wild-type and -deficient MEFs; scale bar $=10 \mu \mathrm{m}$. (B) Quantification of microtubule aster projections in the presence of nocodazole (time $=0 \mathrm{~min}$ ) and after release for 3 and $6 \mathrm{~min}$, respectively (at least 10 cells counted in each condition; ${ }^{*} p<0.05$; and error bars show SD for the measured cells)

The Rho family of small GTPases is involved in several fundamental cellular processes, such as proliferation and motility [34]. ROCK2 is a serine/threonine kinase that is highly expressed in brain and regulates actin and microtubule dynamics [34]. Thus, ROCK2 plays a key role in cell migration, differentiation and mitotic phases such as cytokinesis [34,35]. Consistent with these observations, decreased ROCK2 expression has been correlated with aberrant cytokinesis in previous studies [28]. While ROCK2 is usually found in the cytoplasm, it also co-localizes with PLK1 at the cleavage furrow during cytokinesis [28]. Indeed, in vitro studies have suggested that PLK1 phosphorylates and activates ROCK2 to regulate the actomyosin ring contraction that is required for cleavage furrow ingression during cytokinesis [28]. Thus, PLK1 and TACC3 may not have a direct role in the neuropathology of TSC, but their effectors and/or regulators (ROCK2, as shown in this study, and chTOG) are proposed to be altered in tuber development. Collectively, decreased expression of ROCK2 downstream of loss of tuberin has the potential to disrupt mitosis and induce binucleated cells. However, unlike the cases of tuberin-deficient MEFs [12] and human tubers (shown here), the stable silencing of TSC2 expression in human BJ fibroblasts was not sufficient

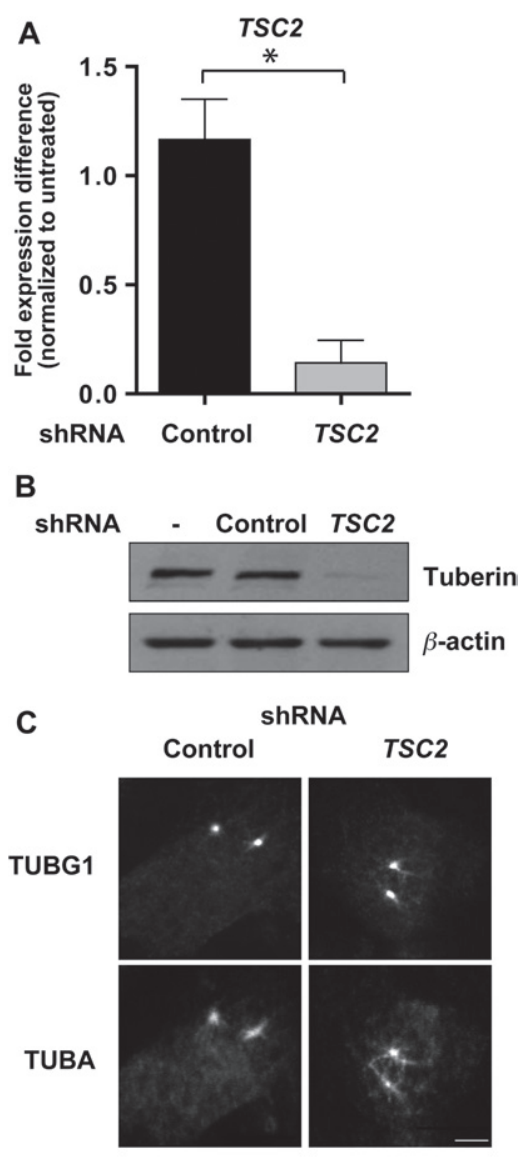

D

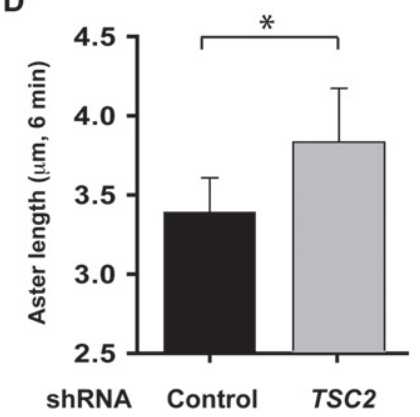

Figure 6. Stable depletion of TSC2 expression in human fibroblasts increases microtubule regrowth. (A) Human BJ fibroblasts with stable shRNA-mediated silencing of TSC2 or expressing a control (non-hairpin construct) were profiled for expression of TSC2, using qRT-PCR assays. Cells silenced for TSC2 show a $\sim 70 \%$ reduction in expression of this gene; error bars indicate SD $(n=3)$. (B) Decreased expression of tuberin in TSC2-silenced cells relative to control; actin served as a loading control. (C) Representative images of microtubule regrowth in control non-hairpin and TSC2-silenced fibroblasts are shown; scale bars $=10 \mu \mathrm{m}$. (D) Significant $(p<0.05)$ increase in aster microtubule length revealed in fibroblasts silenced for TSC2 expression; error bars indicate SD $(n=3)$

to induce binucleated cells or disrupt nuclear envelope morphology. This contradiction may be due to incomplete depletion of tuberin in TSC2-silenced BJ fibroblasts (ie $60-70 \%$ depletion). In addition, it remains to be elucidated whether similar alterations are linked to hamartin deficiency. The previous identification of an excess of binucleated cells and cytokinetic abnormalities in hamartin-deficient cell cultures $[12,24]$ suggests convergence on the same mechanism. 
A

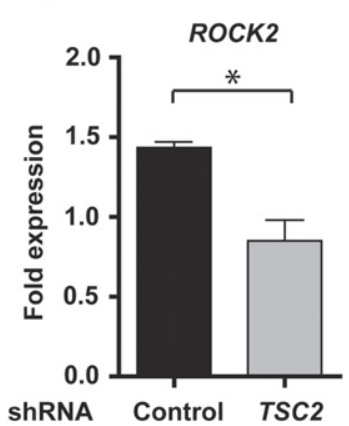

B

ShRNA

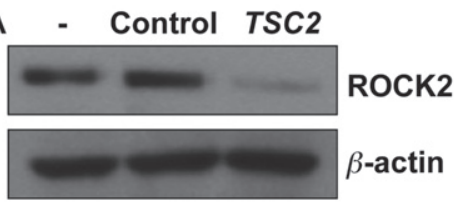

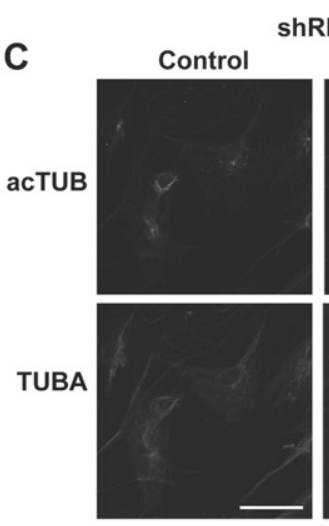

ShRNA

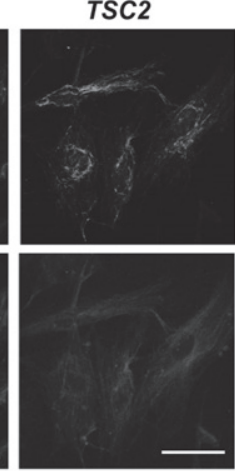

D

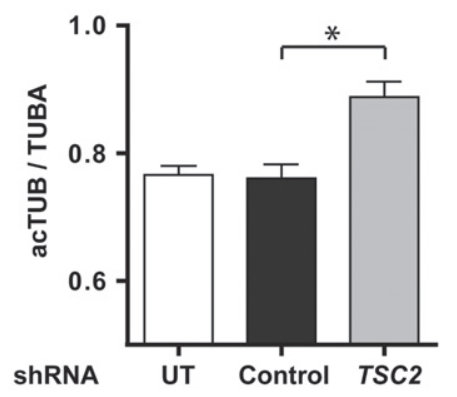

E

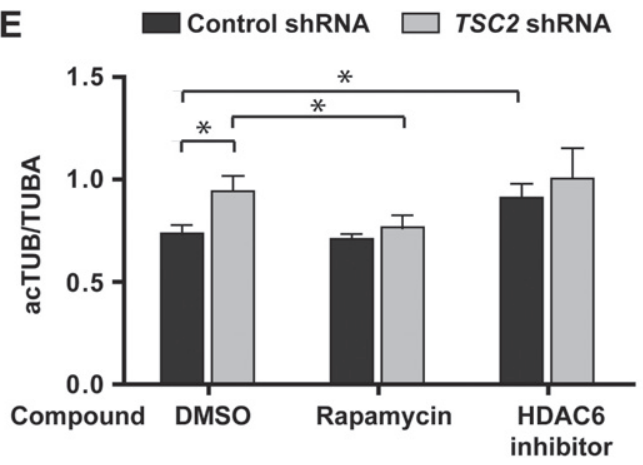

Figure 7. The ROCK2-TPPP1-HDAC6 pathway is affected in TSC2-silenced fibroblasts. (A) Expression of ROCK2 was measured using qRT-PCR assays from cDNA isolated from fibroblasts with stable silencing of TSC2 expression, non-hairpin control or untreated cells. Decreased expression of ROCK2 ( $p<0.05)$ was observed in TSC2-silenced cells compared to non-hairpin control. The expression in non-hairpin treated control and TSC2-silenced cells was normalized to the expression of ROCK2 in untreated cells $(n=2)$. (B) Decreased expression of ROCK2 in TSC2-silenced cells relative to control; actin served as a loading control. (C) Levels of acTUB were measured in control, non-hairpin-treated and TSC2-silenced fibroblasts by immunofluorescence staining; representative images of acTUB and total tubulin (TUBA) are shown; scale bars $=20 \mu \mathrm{m}$. (D) Levels of acTUB and total tubulin were analysed by confocal microscopy in untreated (UT), control-treated and TSC2-silenced cells. The levels of acTUB were normalized to that of total tubulin, and a significant increase was observed in TSC2-silenced cells compared to control $(p<0.05 ; n=3)$. (E) Untreated (UT, not shown), control non-hairpin-treated and TSC2-silenced cells were exposed to rapamycin, a specific HDAC6 inhibitor, or a vehicle (DMSO) control for $16 \mathrm{~h}$ and acTUB was quantified with confocal microscopy. Relative to vehicle treatments, rapamycin treatment significantly $(p<0.05)$ reduced the levels of acTUB in TSC2-silenced cells, but not in controls. Relative to vehicle treatments, treatment with the HDAC6 inhibitor significantly $(p<$ $0.05)$ increased the levels of acTUB in controls, but not in TSC2-silenced cells $(n=3)$

Other functions of ROCK2 include the regulation of microtubule stability through a downstream substrate, TPPP1. ROCK2 phosphorylates TPPP1, which prevents the interaction between TPPP1 and HDAC6, and in turn enables HDAC6 to deacetylate and destabilize microtubules [26]. Therefore, decreased expression or activity of ROCK2 should sequester HDAC6, and elevate microtubule acetylation in the cell. Our results in human fibroblasts are consistent with this mechanism of action, showing that loss of tuberin attenuated ROCK2 expression and augmented microtubule acetylation to a level that was obtained with HDAC6 inhibition in parental and control-treated cell lines. Interestingly, changes to the ROCK2-TPPP1-HDAC6 signal transduction pathway modulate cellular migration [26], which could provide an explanation for the impairment of axon differentiation and cell migration in tuberin-deficient mouse and rat hippocampal neurons [8]. Together, our findings support the hypothesis that fundamental changes in microtubule biology underlie TSC neuropathology and highlight that these changes may arise through the specific disruption of ROCK2 signalling.

\section{Acknowledgements}

We wish to thank the TSC patients and their families for their valuable participation, and the Asociación Nacional de Esclerosis Tuberosa for their support. We also wish to thank Aristotelis Astrinidis for scientific discussions, Enrique Fernández-Taboada for technical assistance, and Elena Goncharova and Aristotelis Astrinidis for providing reagents. The research was supported by the Generalitat de Catalunya (Grant Nos 2009-SGR283 and 2009-SGR293), Red Temática de Investigación Colaborativa en Cáncer (Grant Nos RD06/0020/1050 and RD12/0036/0008), the Seventh Framework Programme of the European Commission (Grant No. 278486, DEVELAGE), a Child and Youth Health, New Investigator Award from the SickKids Foundation and the Institute of Human Development Child and Youth Health-CIHR (Grant No. NI11- 005), a Paediatric brain tumour grant from the Michael Cuccione Foundation for Childhood Cancer Research, and the Spanish Ministry of Health 'Instituto de 
Salud Carlos III Fondo de Investigación Sanitaria' (Grant Nos CA10/01474, PI10/01422, PI12/01528 and PI13/00285).

\section{Author contributions}

IF, CAM and MAP conceived the study; PM, JC, LGB, HC, MC, NG, HA, JJ, MS, MN, IA, AR and CL provided or prepared tissues, carried out experiments and analysed data; and PM, CAM and MAP wrote the manuscript. All authors had final approval of the submitted manuscript.

\section{Abbreviations}

acTUB, acetylated tubulin; GSEA, gene set expression analysis; GO, Gene Ontology; MEFs, mouse embryonic fibroblasts; mTOR, mechanistic target of rapamycin; PLK1, polo-like kinase 1; pS6, phospho-Ser235-236 S6 ribosomal protein; qRT-PCR, quantitative reverse transcription-polymerase chain reaction; ROCK2, Rho-associated coiled-coil containing kinase 2; TACC3, transforming acidic coiled-coil containing protein 3; TSC, tuberous sclerosis complex; TUBA, $\alpha$-tubulin; TUBG1, $\gamma$-tubulin.

\section{References}

1. Crino PB, Nathanson KL, Henske EP. The tuberous sclerosis complex. N Engl J Med 2006; 355: 1345-1356.

2. Mizuguchi M, Takashima S. Neuropathology of tuberous sclerosis. Brain Dev 2001; 23: 508-515.

3. Crino PB. The pathophysiology of tuberous sclerosis complex. Epilepsia 2010; 51(suppl 1): 27-29.

4. Zoncu R, Efeyan A, Sabatini DM. mTOR: from growth signal integration to cancer, diabetes and ageing. Nat Rev Mol Cell Biol 2011; 12: $21-35$.

5. Gomez MR, Samson JR, Whittemore VH. Tuberous Sclerosis Complex, 3rd edn. Oxford University Press: New York, 1999; 3-9.

6. Orlova KA, Crino PB. The tuberous sclerosis complex. Ann NY Acad Sci 2010; 1184: 87-105.

7. Choi YJ, Di Nardo A, Kramvis I, et al. Tuberous sclerosis complex proteins control axon formation. Genes Dev 2008; 22: 2485-2495.

8. Magri L, Cambiaghi M, Cominelli M, et al. Sustained activation of mTOR pathway in embryonic neural stem cells leads to development of tuberous sclerosis complex-associated lesions. Cell Stem Cell 2011; 9: 447-462.

9. Way SW, McKenna J, 3rd, Mietzsch U, et al. Loss of $T s c 2$ in radial glia models the brain pathology of tuberous sclerosis complex in the mouse. Hum Mol Genet 2009; 18: 1252-1265.

10. Goto J, Talos DM, Klein P, et al. Regulable neural progenitor-specific Tsc1 loss yields giant cells with organellar dysfunction in a model of tuberous sclerosis complex. Proc Natl Acad Sci USA 2011; 108: E1070-1079.

11. Tanwar PS, Kaneko-Tarui T, Zhang L, etal. Altered LKB1/AMPK/TSC1/TSC2/mTOR signaling causes disruption of Sertoli cell polarity and spermatogenesis. Hum Mol Genet 2012; 21: $4394-4405$.

12. Gómez-Baldó L, Schmidt S, Maxwell CA, et al. TACC3-TSC2 maintains nuclear envelope structure and controls cell division. Cell Cycle 2010; 9: 1143-1155.
13. Astrinidis A, Senapedis W, Henske EP. Hamartin, the tuberous sclerosis complex 1 gene product, interacts with polo-like kinase 1 in a phosphorylation-dependent manner. Hum Mol Genet 2006; 15: 287-297.

14. Bonnet CS, Aldred M, von Ruhland C, et al. Defects in cell polarity underlie TSC and ADPKD-associated cystogenesis. Hum Mol Genet 2009; 18: 2166-2176.

15. DiBella LM, Park A, Sun Z. Zebrafish Tsc1 reveals functional interactions between the cilium and the TOR pathway. Hum Mol Genet 2009; 18: 595-606.

16. Hartman TR, Liu D, Zilfou JT, et al. The tuberous sclerosis proteins regulate formation of the primary cilium via a rapamycin-insensitive and polycystin 1-independent pathway. Hum Mol Genet 2009; 18: $151-163$.

17. Chen Z, Indjeian VB, McManus M, et al. CP110, a cell cycle-dependent CDK substrate, regulates centrosome duplication in human cells. Dev Cell 2002; 3: 339-350.

18. Maxwell CA, Benitez J, Gómez-Baldó L, et al. Interplay between BRCA1 and RHAMM regulates epithelial apicobasal polarization and may influence risk of breast cancer. PLoS Biol 2011; 9: e1001199.

19. Mohan P, Castellsague J, Jiang J, et al. Genomic imbalance of HMMR/RHAMM regulates the sensitivity and response of malignant peripheral nerve sheath tumour cells to aurora kinase inhibition. Oncotarget 2013; 4: 80-93.

20. Boer K, Crino PB, Gorter JA, et al. Gene expression analysis of tuberous sclerosis complex cortical tubers reveals increased expression of adhesion and inflammatory factors. Brain Pathol 2010; 20: 704-719.

21. Blake JA, Harris MA. The Gene Ontology (GO) project: structured vocabularies for molecular biology and their application to genome and expression analysis. Curr Protoc Bioinformatics 2008; 7: Unit 7.2.

22. Subramanian A, Tamayo P, Mootha VK, et al. Gene set enrichment analysis: a knowledge-based approach for interpreting genome-wide expression profiles. Proc Natl Acad Sci USA 2005; 102: $15545-15550$.

23. Normand G, King RW. Understanding cytokinesis failure. Adv Exp Med Biol 2010; 676: 27-55.

24. Toyoshima M, Ohno K, Katsumoto T, et al. Cellular senescence of angiofibroma stroma cells from patients with tuberous sclerosis. Brain Dev 1999; 21: 184-191.

25. Doxsey S, Zimmerman W, Mikule K. Centrosome control of the cell cycle. Trends Cell Biol 2005; 15: 303-311.

26. Schofield AV, Steel R, Bernard O. Rho-associated coiled-coil kinase (ROCK) protein controls microtubule dynamics in a novel signaling pathway that regulates cell migration. J Biol Chem 2012; 287: 43620-43629.

27. Schofield AV, Gamell C, Suryadinata R, et al. Tubulin polymerization promoting protein 1 (Tppp1) phosphorylation by Rho-associated coiled-coil kinase (rock) and cyclin-dependent kinase 1 (Cdk1) inhibits microtubule dynamics to increase cell proliferation. J Biol Chem 2013; 288: 7907-7917.

28. Li J, Wang J, Jiao H, et al. Cytokinesis and cancer: Polo loves ROCK'n' Rho(A). J Genet Genom 2010; 37: 159-172.

29. Lowery DM, Clauser KR, Hjerrild M, et al. Proteomic screen defines the Polo-box domain interactome and identifies Rock2 as a Plk1 substrate. EMBO J 2007; 26: 2262-2273.

30. Nogales-Cadenas R, Abascal F, Diez-Perez J, et al. CentrosomeDB: a human centrosomal proteins database. Nucleic Acids Res 2009; 37: D175-180.

31. Sole X, Bonifaci N, Lopez-Bigas N, et al. Biological convergence of cancer signatures. PLoS One 2009; 4: e4544.

32. Al-Bassam J, Chang F. Regulation of microtubule dynamics by TOG-domain proteins XMAP215/Dis1 and CLASP. Trends Cell Biol 2011; 21: 604-614. 
33. Kuijpers M, Hoogenraad CC. Centrosomes, microtubules and neuronal development. Mol Cell Neurosci 2011; 48: 349-358.

34. Riento K, Ridley AJ. Rocks: multifunctional kinases in cell behaviour. Nat Rev Mol Cell Biol 2003; 4: 446-456.
35. Lock FE, Hotchin NA. Distinct roles for ROCK1 and ROCK2 in the regulation of keratinocyte differentiation. PLoS One 2009; 4: e8190.

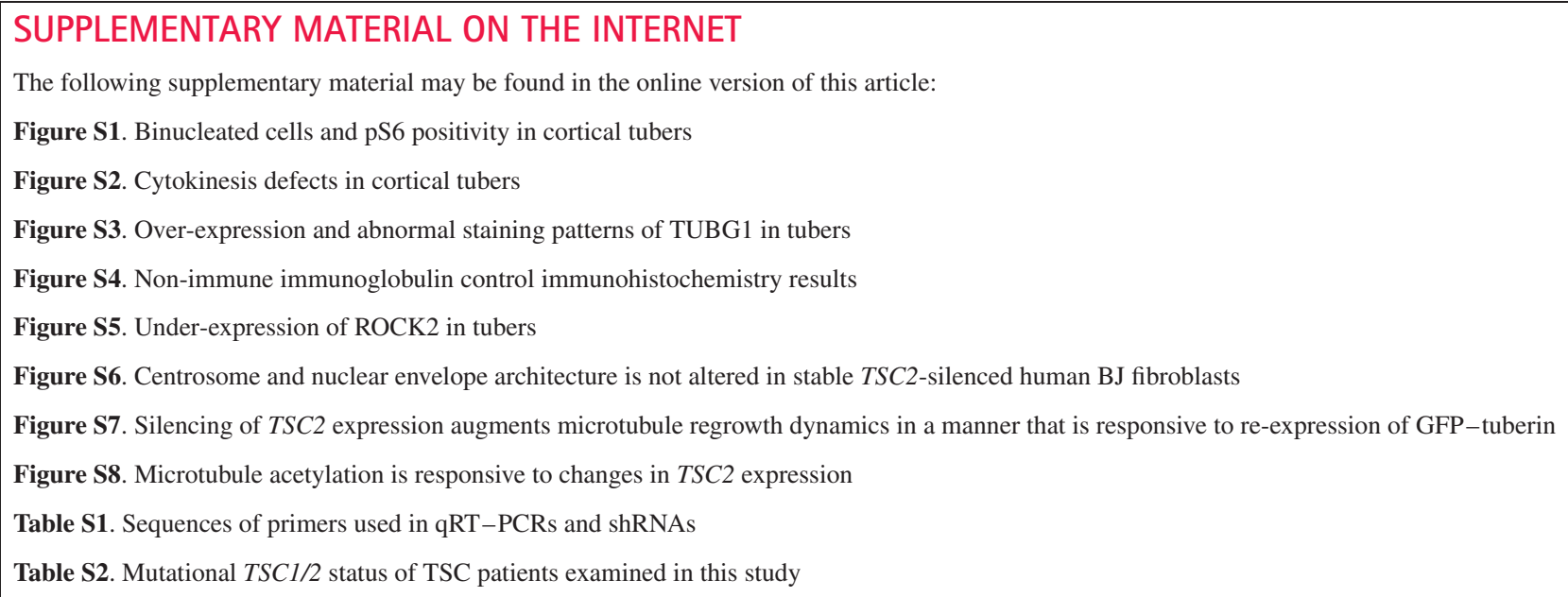

\title{
25 Years ago in the Journal of Pathology...
}

\author{
Morphometric analysis of nuclear features, ploidy status, and staging in \\ rectal carcinoma \\ Dr. Christopher D. Carter, Sandy C. Weeks, Lynn R. Jarvis and Richard \\ Whitehead
}

The ultimobranchial gland and congenital thyroid abnormalities in man

Professor E. D. Williams, C. E. Toyn and H. R. Harach

Microglial cells in human brain have phenotypic characteristics related to possible function as dendritic antigen presenting cells

Dr. J. Lowe, K. A. Maclennan, D. G. Powe, J. D. Pound and J. B. Palmer

To view these articles, and more, please visit: www.thejournalofpathology.com

Click 'ALL ISSUES (1892 - 2011)', to read articles going right back to Volume 1, Issue 1.

\section{The Journal of Pathology Understanding Disease}

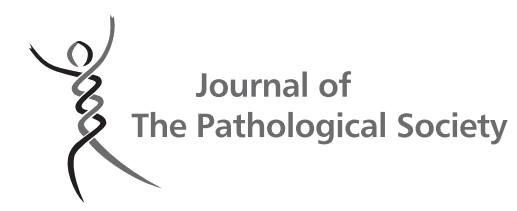

